\title{
A Radar Visualization System Upgrade
}

\author{
Hector N. Acosta, Marcelo A. Tosini, María C. Tommasi, Lucas Leiva
}

INTIA Research Institute, INCA Group, Buenos Aires, Argentine

Email: \{nacosta, mtosini, ctommasi, 1leiva\}@exa.unicen.edu.ar

Received September $9^{\text {th }}, 2009$; revised September 12 ${ }^{\text {th }}, 2009$; accepted December $1^{\text {st }}, 2009$.

\begin{abstract}
This work develops a system to visualize the information for radar systems interfaces. It is a flexible, portable software system that allows to be used for radars that have different technologies and that is able to be adapted to the specific needs of each application domain in an efficient way. Replacing the visualization and processing units on existing radar platforms by this new system, a practical and inexpensive improvement is achieved.
\end{abstract}

Keywords: Radar, Data Visualization, Signal Processing, Signal Pattern Recognition

\section{Introduction}

Conceptually, a radar system consists of five components: a transmitter, a receiver, an amplifier, an analyzer and a visualizer. This work describes the development of a software system to replace the last two components, in order to bring up to date the present services of different radars, air traffic control radars, tactical radars, navigation radars for military or civil use, both naval and aeronautic.

The analyzer of a radar system has to obtain the desired information from the received signals, and to determine if the reflections got through the antenna corresponds to targets of interest for the system. The analyzing components of modern systems carry out a great number of functions that allow to synthetize the desired information in an efficient and simple way, as noise minimize, prediction of target course, objective identification.

The visualizer component has to show the information processed by the analyzer on a screen or a display. This can be done in different ways depending on the needs of the system. For example, search or surveillance radars that cover $360^{\circ}$ use to present information as a Plan Position Indicator (PPI) (Figure 1), which show targets in a polar form centered in the radar position, whereas tactical Air-to-Air radars, use presentations as "B-Scope" (Figure. 2 ), which put the different targets on the display according to distance information (vertical line) and azimuth (horizontal line) [1].

Radars are very expensive electronic equipment and as every technologic tool must be depreciated very soon because it should be replaced by new, more complex models. Companies are reluctant to change equipment, but operators intend to be up to date with new technology, by getting constant training. This situation puts a challenge between equipment being used and operators training. As a partial solution, this article introduces and analyzes the use of a generic parametrizable system that permits to update the radar in a rather practical and economical way. This approach proposes using the existing technologic platform and adding new functionality to provide services that approximate desired solutions avoiding expensive and sometimes inviable alternative of whole replace of the equipment.

In the following sections the design and development work of a radar signals visualization system is described. Then, the implementation of the system is given in detail as a case study adapted to a specific technological platform.

\section{Architectural Design}

A radar system is composed of five components: a transmitter, a receiver, an amplifier, an analyzer and a visualizer. As the system to be developed must replace and enhance the analyzing and visualizing components, it's important to describe some characteristics of the other components, in order to establish the operative conditions of the system and, thus, its requirements. The architectural design of the system is based on a detailed analysis of the functional and non-functional characteristics required.

\subsection{Functional Characteristics}

The system must be able to process signals that come from different devices, as well as from other software and/or hardware components. Signals processed by the system may be classified into the following types: 


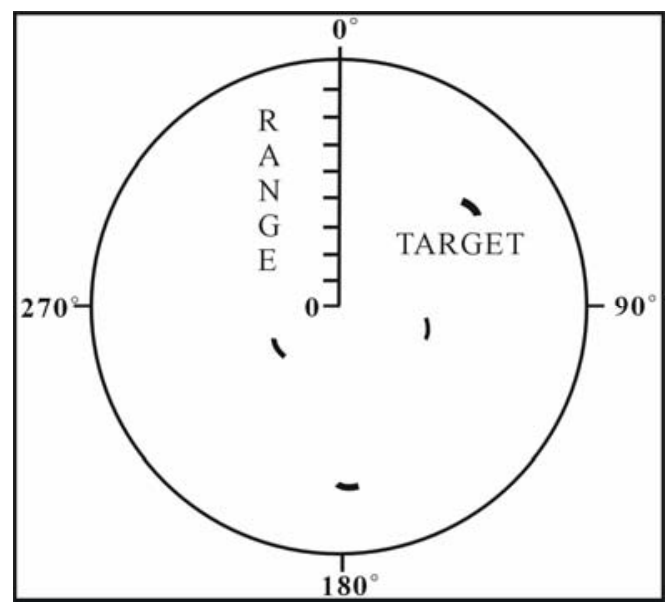

Figure 1. Presentation "Plan Position Indicator" (PPI) 1 CONICET

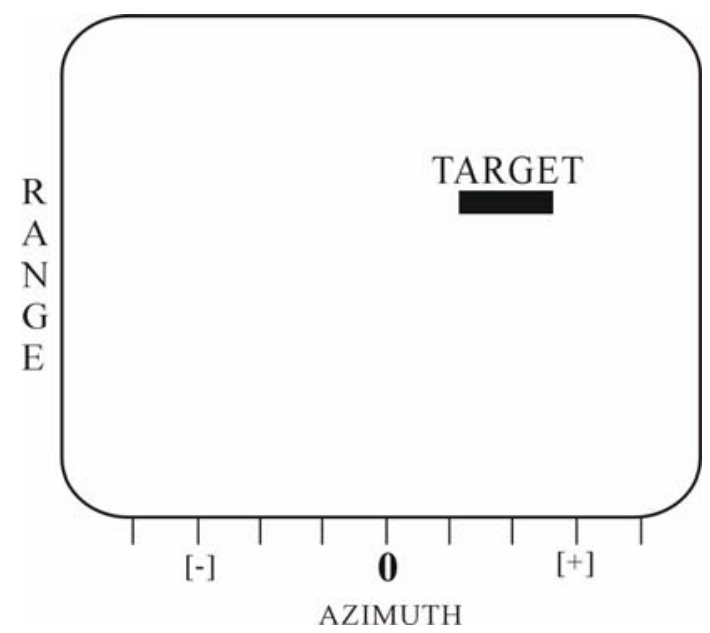

Figure 2. "B-Scope"

- Analogic and/or digital signals coming from the radar (antenna position, echo receiving, synchronization, operation modes of the radar).

- Analogic and/or digital signals coming from hardware devices external to the radar (GPS, timers, course and attitude indicators).

- Digital signals coming from independent software components that can communicate with the system for specific purposes (targets detection, geographic information system, navigation system, anti collision system).

- Signals coming from manual controls of the support device of the system (mainly keyboard and joystick).

Signals from the radar device are variable and have different characteristics. First of all, it is necessary to know the reflections values obtained from the antenna of the radar. Besides, those data must be processed along with the antenna position (which depends on the radar type and the operation modes provided) and synchronization information between times of sending and receiv- ing, in order to achieve an adequate representation of the zone explored by the radar. Besides there are signals originating from specific functionalities provided by the radar, for example, targets tracking functions, used in air traffic control radar systems or tactical radars for military uses.

Often, modern radar systems communicate with other devices for specific purposes. In meteorological or air traffic control radars is very usual, for example, add to the information detected by the radar geographic information or satellital images of the explored area. In radars installed on ships or aircrafts it is of the utmost importance to have georeferencial navigation information, usually provided by some device or external system (GPS, for example). This is why, it is very important when designing and developing a system with these characteristics to have in mind that input signals can be distinct in nature and source.

Finally, it must be taken into account that modern radars have different modes of operation, in each of which the functioning of the device can be changed, altering, for example, the way the antenna moves, and thus, the exploration zone, or simply the information on the display. These changes in functioning parameters must be modified by the operator, through system controls.

As the result of signals processing, the system must show information about the objects detected by the radar on a display. This information depends on the application domain, nevertheless it can be generalized, so that the system presents a set of "objects" and "attributes", updating the changes of the objects and selecting those attributes that can be ignored or pointed out for presentation.

With regard to functionalities of interface, the system must provide a multifunction visualizing unit (MFD Multi Function Display) to show graphical presentations flexible and easy to modify and adapt to the user's needs. It is essential for this purpose to be able to define different kinds of presentations and configuring their characteristics. So, the system must have ability to manage the iconography and graphical and textual information to visualize in different contexts in flexible way.

\subsection{Non-Functional Characteristics}

Basically, this system must carry out the acquisition, interpretation and presentation of information in real time. There are conditions that critically affect the performance of the system, which must be considered in the architectural design.

The system must fulfill hard performance requirements, reacting to user orders and updating all detected changes of the information to show on MFD with celerity. The display is an accurate representation of the zone being explored.

The system must also be updated easily in order to 
adapt to changes, both regarding to the application domain and users' needs. It also must adapt in a simple way to process signals coming from new hardware devices or software components, to modify the algorithms for signal processing, to change or add presentation of information formats, among other important functionalities.

Another basic aspect is the technological independence. The software embedded in the system must be based on open source code (ANSI C) and have all the sources of every level of coexisting software (operating system, drivers, fonts, interfaces).

Finally, it is relevant to consider that the system must be implemented on a platform providing a high level of performance and synchronization.

\subsection{Software Architecture}

The characteristics desired in software architecture must ensure an easy evolution of the system. That evolution must consider changes in time, the impact of these changes in the different components and/or devices interacting with the system and the independence of functionalities of processing and visualization of the underlying technological platform [2].

Considering these objectives and the functional and non-functional characteristics above described, a software architecture based on three components was designed: The first is the acquisition component, which collects information that allows to control different devices and to gather analogical and digital signals. The second is the analyzing component, developed by different interpretation algorithms that allow identifying relevant information to select and sort the elements that must be visualized. The third is the visualizing component that allows information under different configurations to be charted. Thus, two components of the present radar (analyzer and visualizer) are replaced by a modern module composed by three components: acquisitor, analyzer and visualizer.

\subsubsection{Acquisition Component}

It implements the reading of the different signals that the system must process. It is mainly composed of control drivers for different boards and hardware devices of input signals.

The signals that must be processed come from different sources (radar antenna, timers, GPS, navigation system, manual controls), therefore, the system must synchronize the reception of signals.

\subsubsection{Analyzing Component}

It processes and analyzes a set of signals with different characteristics; some of which could need storage of historic information (tracking algorithms and path predictions, study of frequency for target recognizing). It receives a variable set of data as input that represent the different changes of input signals, and produces a set of data representing changes in the information to display on the MFD.

The functions that the system must implement depend directly on the application domain. There are many signal processing techniques for radar systems development on different domains.

Techniques based on Fourier Analysis have important application in many radar systems, a number of computational calculus methods have been developed as the Cooley-Tukey technique used for spectrum analysis and digital signal processing [1]. Generally, techniques based on Fast Fourier Transform (FFT) are used in pulse-doppler radars, radars for tracking mobile targets, vibration analysis on laser radar systems [3].

\subsubsection{Pattern Recognition Component}

Algorithms for pattern recognition based on artificial intelligence techniques are also used, neural nets and fuzzy logic [4-6]. On last generation radars, pattern recognition is used to classify known echos in different categories.

For tactical radars it is important to consider target tracking: only one target, multiple targets and "false alarms". These approaches use probabilistic and estimation techniques, Kalman filters, fuzzy logic, neural networks [7].

In this case, a pattern recognition system based on RBF networks is implemented

\subsubsection{Visualizing Component}

It implements all the functionalities of MFD, without processing or transformation of the received information, it only provides functions to configure graphic presentations and shows information for the operator on the display.

There are many kinds of presentations according to different application domain. For example, the iconography used to describe an objective on the display is different for military purposes radars or for aerial navigation radar, civil or military.

\section{Signals Acquisition}

The acquisition component was designed to provide functions of signal acquisition, data conversion and synchronization of processes to read information.

It is composed of the control drivers of the hardware devices involved and software for reading data. It interacts with the different components obtaining relevant information and carries out all needed transformation of data required by the system. Therefore, analogic signals are digitalized using analog/digital converter boards, both if they are connected to the PC or to the FPGA. This component also implements all conversion of data necessary for the processing component to receive an standardized input set of information, according to a previously defined format. 


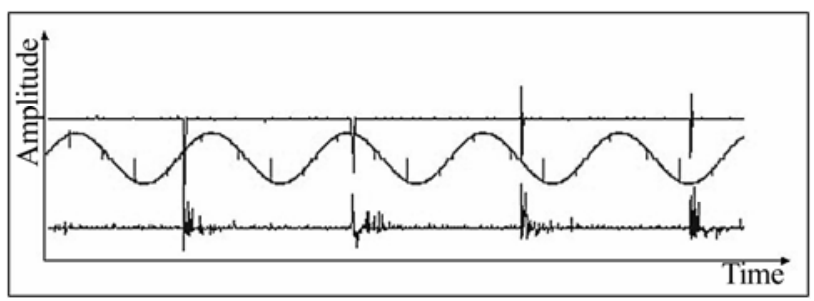

Figure 3. Signal L1 (upper), Signal H1 (middle) and Signal P1 (lower)

The following is an example of the characterization of some signals studied on the radar commercialized as Cyrano (Figure 3):

General Synchro signal: (L1). Voltage range: +/- 40 Volts. Chain of pulses with $500 \mathrm{~ms}$ of separation between pulses and width of pulse of $2 \mathrm{~ms}$. Each pulse indicates the beginning of a phase of issue of the radar antenna. The system produces 2000 pulses a second.

Video indicator signal: (P1). Voltage range: +/- 5 Volts. This signal is the output of the stage of intermediate frequency that forms the signal coming from the radar antenna. As the radar produces 2000 pulses a second, a signal of 1500 cycles for each pulse of the antenna is obtained. Frequency: $3 \mathrm{Mhz}$. Noise level: When not transmitting it is 2 Volts. When transmitting it is $+/-200$ $\mathrm{mV}$.

Antenna Y scanning signal: (H1). Voltage range: +/37 Volts. This signal indicates the horizontal shift of the radar antenna. Values measured in radar of $37 \mathrm{~V}$ and 530 $\mathrm{Hz}$, with $180^{\circ}$ for $+60^{\circ}$ and $0^{\circ}$ for $-60^{\circ}$ antenna position.

In Figure 3, a section of exploration of 4 cycles is observed. On signal L1, the pulses of synchronization determine the beginning of the cycle. As for the signal $\mathrm{H} 1$, it can be seen that for each cycle of L1, there is approximately a complete cycle of $\mathrm{H} 1$, which indicates the horizontal position of the radar antenna at the beginning and at the end of each cycle. Finally, P1 describes the video information received by the radar in each cycle showing some nearby contacts and many contacts of little intensity (noise) along the rest of the section.

\section{Signals Processing}

\subsection{General Description}

The Analyzing Component was designed to analyze the different input signals in real time, calculating the values of the "elements" or "objectives" of the presentations and sending this information to the Visualizing Component

To compute the data of some objectives it is necessary to analyze the information coming from several signals. The information of some signals must be used in the calculation of several objectives, so any change in the input signals implies recalculate the data of all the objectives affected by that change. This mechanism is implemented through a two dimensional matrix containing the infor- mation of dependence among objectives to be charted and input signals. This "Matrix of Dependencies" contains in each cell a pointer to a function; if there is a dependence between signal and objective a function is referenced, which calculates the information corresponding to the objective and sends it to the Visualizing Component, otherwise, the reference is nil.

The input data received from the Acquisition Component and the output data sent to the Visualizing Component are transmitted by structures of communication called «SignalPipe», implemented by sockets of TCP protocol. These structures were implemented to provide a standard interface for the communication among the components.

Before being processed, the signals are filtered in order to reduce the white noise coming from conversion. To do so, a Kalman filter is used [8]. This filter reduces the chance of making mistakes while recognizing patterns.

The time update equations are defined as:

$$
\begin{gathered}
\hat{x}_{k}^{-}=\hat{x}_{k-1} \\
P_{k}^{-}=P_{k-1}+Q
\end{gathered}
$$

and the measurement update equations are:

$$
\begin{gathered}
K_{k}=\frac{P_{k}^{-}}{P_{k}^{-}+R} \\
x_{k}=x_{k}^{-}+K_{k}\left(z_{k}+x_{k}^{-}\right) \\
P_{k}=\left(1-K_{k}\right) P_{k}^{-}
\end{gathered}
$$

Thus, the signals are filtered reducing significantly their noise. (Figure 4)

\subsection{Component Implementation}

The Analyzing Component in charge of the processing of signals (Figure 5) is composed of:

A structure of information storage refered to the specified set of input signals.

A set of calculus algorithms of all the attributes and objectives that plot the defined presentations.

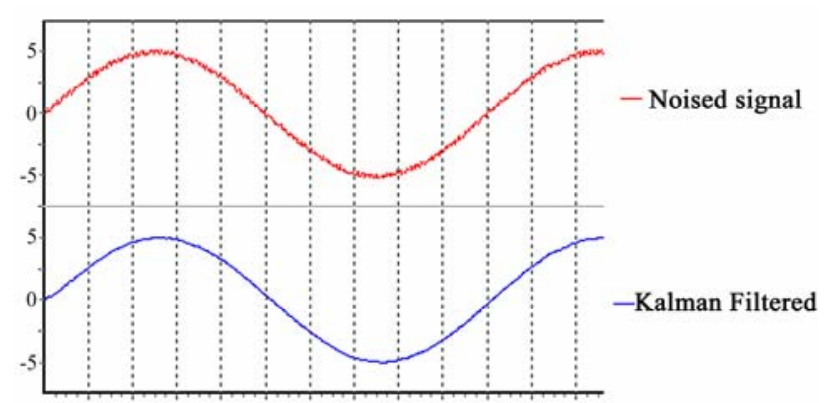

Figure 4. White noise reduction by Kalman filter 


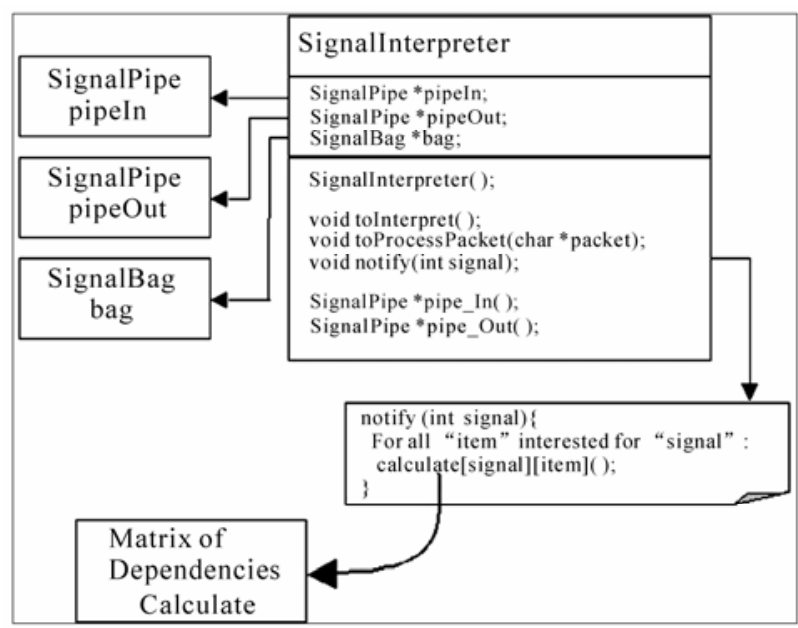

Figure 5. Implementation diagram of the Analyzing Component

A structure of dependences between input signals and output objectives.

References to communication structures with the Acquisition Component and the Visualizing Component.

The structure of signal storage implemented in the class «SignalBag» keeps all the necessary information for different signals during runtime. Every time a change of a signal is received the structure is updated and the affected objectives are notified. Internally, this structure has a buffer of storage for each signal. These buffers are of variable length (according to the characteristics of each signal) and can be updated and easily examined both to incorporate the new data and to use the stored data to calculate the visualization information.

The notification process invocates the calculus routines of the affected objectives and the sending of the updated data to the Visualizing Component. To carry out this task, the component has a structure to establish which objectives depend on which signals. This structure "Matrix of Dependencies" and contains references to the processing algorithms for different objectives. These algorithms are run by the «calculate» method.

The references to the structures of communication with the Acquisition and Visualizing Components are called «pipeIn» and «pipeOut».

The class «SignalInterpreter» is the main class of the component and implements the mechanism of signal processing. It is realized by the method «toInterpret» (Figure 6). The method «toInterpret» implements the main function of the component, which receives from the input pipe «pipeIn» the changes in the signals. For each packet received the method «toProcessPacket» is invocated, it updates the values of the signals in the "bag" structure and invocates the functions of calculus of the objectives affected by the change, which notify their results to the output pipe «pipeOut».

\subsection{Data Sending and Receiving}

The sending and receiving of information with the Acquisition and Visualizing Components is carried out according to the conventions defined by the structure of communication «SignalPipe», where the messages are textual and have a format of 3-ary ELEMENT-ATTRIBUTE-VALUE. When the messages to be sent correspond to 2-ary the third field is completed with the value zero ("0").

The messages received by the component use the format of two elements, where the field ELEMENT corresponds to an identifier of the input signal and the field VALUE corresponds to the numerical value. Messages sent to the Visualizing Component use the format of three elements where the field ELEMENT corresponds to an identifier of the objective in the presentation, the field ATTRIBUTE corresponds to a characteristic of the objective and the field VALUE corresponds to the numerical value.

\section{Pattern Recognition}

Pattern automatic identification is achieved through the use of classification techniques. Neural networks are one of the approaches to be used.

In the context of neural networks, classification involves to get a function responsible for grouping the data into categories, based on some characteristics. This function is materialized by a neuro-classifier which is trained using different classes of input data together with their categories. A classifier neural network associates an input vector with a represented category producing as output a signal whose value indicates the membership of the

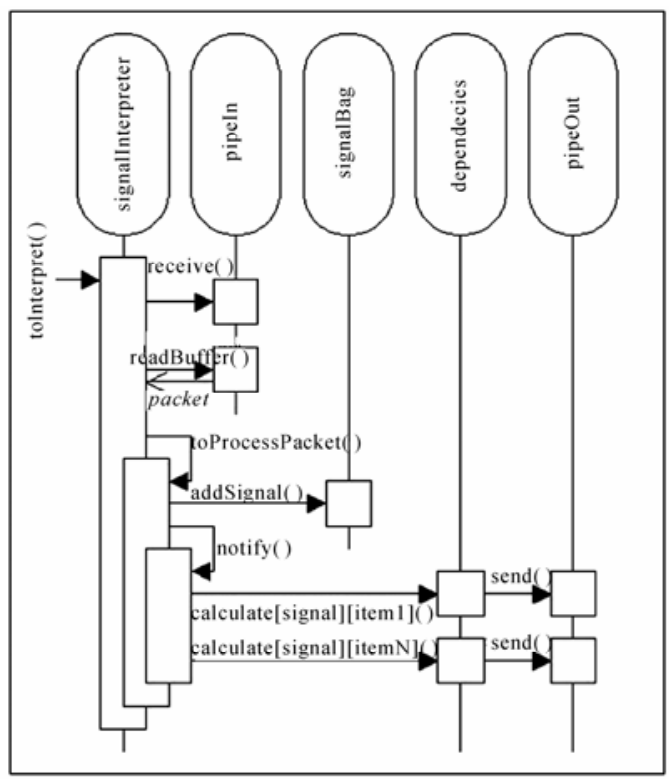

Figure 6. Interaction diagram of method «toInterpret» 
input to the category. The network can also indicate the degree of acceptance of the input to that category; therefore, the output values are not restricted to binary signals.

The RBF networks are generally used as neuro-classifiers and they are well adapted to target identification in radar images [9]. This kind of networks has an input layer composed of branches nodes and a hidden layer for which each node has a special activation function. This function is placed into the central vector of the cluster within the feature space; thus the function has a significant response for those vectors near its center. Each result from the hidden layer has a weight value associated. The output layer is responsible for summing up the products got from the results of the hidden layer, together with their weight values. (Figure 7).

The system presented in this paper has a trained neural network able to classify targets. This net is implemented on a Xilinx's Virtex-4 (xc4vsx25-11-ff668) FPGA [10]. The main advantage of implementation using this platform is the natural parallelism among the networks that is allowed.

The neural network gets the values of $<<$ SignalBag $>>$ taking them as if they were an image. This "image", in a monochromatic format with 12 bits per pixel resolution, is composed by 1500 rows (input data at $3 \mathrm{Mhz}$ ) and 2000 columns corresponding to the whole scan of the antenna $\left( \pm 60^{\circ}\right)$.

A profiling over the video signal (P1) is performed for both axes, to determine the presence of objects. In Figure 8 the result got for a radar image where a B-52 is present is shown.

In the case presented there are two regions to be analyzed: R1 and R2. The first of them corresponds to the location of a target, whereas the second one corresponds to the earth echo.

Region of interest is defined as the sub region that can contain a pattern. For this implementation, the size of the region is fixed $(150 \times 150$ pixels). The center of each region detected during the profiling will also be the center of the region to analyze.

In this case, both regions (Figure 9) are evaluated since the implementation of the classifier allows effective evaluation at a reasonable rate.

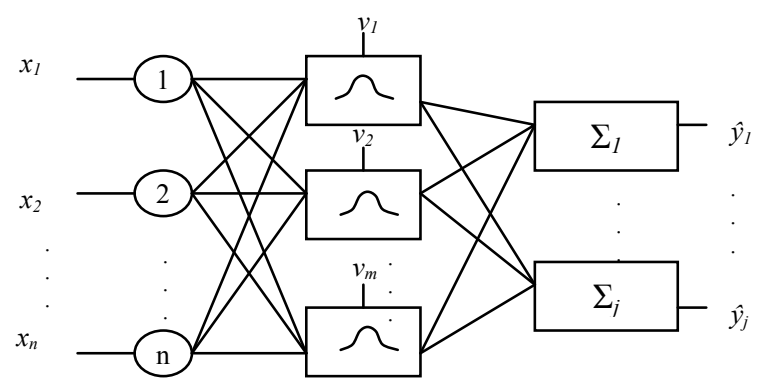

Figure 7. RBF Neural Network

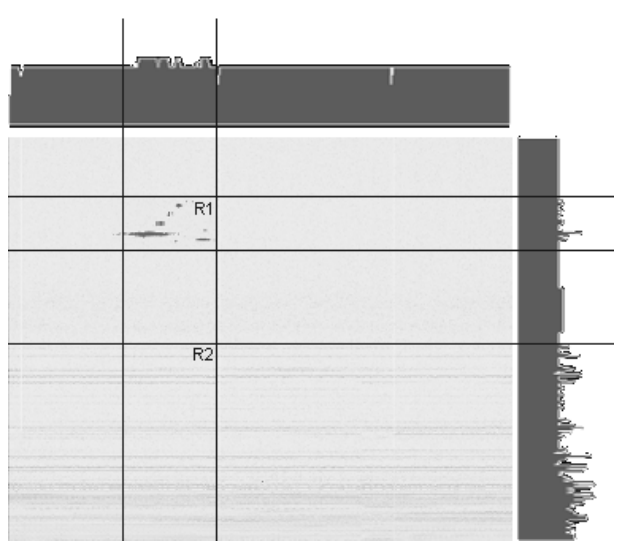

Figure 8. Section profile of a radar image

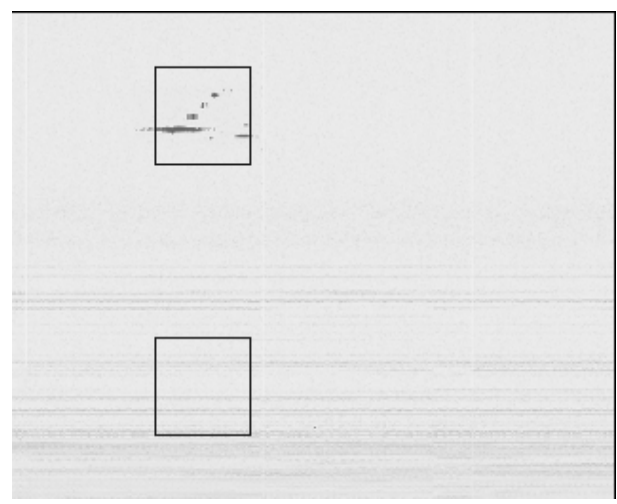

Figure 9. Regions of Interest

Each one of these regions is projected in order to take the most relevant features of the region. Currently the composite profile is being used as characteristic vector since it allows distinction of shapes. However, different techniques for extracting characteristics are being analyzed; these techniques allow more efficient differentiation of patterns (Specially Wavelets). These feature vectors are evaluated by the neural network to classify them.

The hardware neural network architecture does not have on-chip learning; therefore its knowledge is preset. The number of neurons that conforms it depends on the amount and quality of the patterns that allows classification. In this case, the net was trained using 30 patterns corresponding to M-3 class and 30 patterns corresponding to B-52, having as a result a neural network of 25 neurons, with an operational frequency of up to 1000 patterns analyzed per second.

\section{Information Visualization}

\subsection{General Description}

The MFD has, at least, a "view" or "presentation". A view is a set of graphical or textual information presented to the user in a predetermined format.

The Visualizing Component was designed to support 
several parallel views with different formats and conventions that can be modified or replaced in a flexible manner. Two elements were defined for this purpose: «GraphicalItem» «Presentation».

A «GraphicalItem» is any graphical element or textual information that must be presented in the MFD; for example, a contact, the scale rule, information of the scanning of the radar.

A «Presentation» is basically a set of graphicable items. A presentation can be a sinusoidal graph to describe the values of certain signal of the radar, a diagram of the zone explored by the radar, or else a list of numerical data for the operator. Those examples can be different presentations for a same situation. Each kind of presentation is more or less useful according to needs. The present design makes it possible to develop each kind of presentation in an easy and efficient way, because a new presentation only takes three steps: Write an specification of the view in a file, write the code for the graphicable item and set a presentation that includes the specifications and items previously defined.

\subsection{Component Implementation}

The Visualizing Component was developed to handle efficiently graphical and textual information given by «GraphicalItem» and organized as different kinds of screens called «Presentation» whose format is configurable. The visual parameters that represent attributes (color, size, position) of the "Graphicalltem» must be updated in real time.

These functionalities are implemented as follows:

The class «RadarView» is in charge of all functions of the presentation.

The class «GraphicalItem» is in charge of all functions of the items to be displayed.

The formats of the presentations are specified in configuration files to be able to change the presentation with no need to modify or recompile the system.

The changes in the information to show on the display are received through the structure of communication «SignalPipe» with the component of processing «Analyzer».

The class «RadarView» (Figure 10) sets the visualizing format of the view obtaining the information from a previously defined configuration file. The aim of the configuration file is to define the formats of the representation system and the iconography to be used.

In runtime the configuration of the presentation is stored in an inner 2-dimensional structure called «Configuration» which also stores information of the modifications of the presentation when there are changes in the system (change in the mode of operation of the radar, or detection of a specific objective). It is also possible to alter the format of representation in runtime by only updating the matrix from another configuration file.

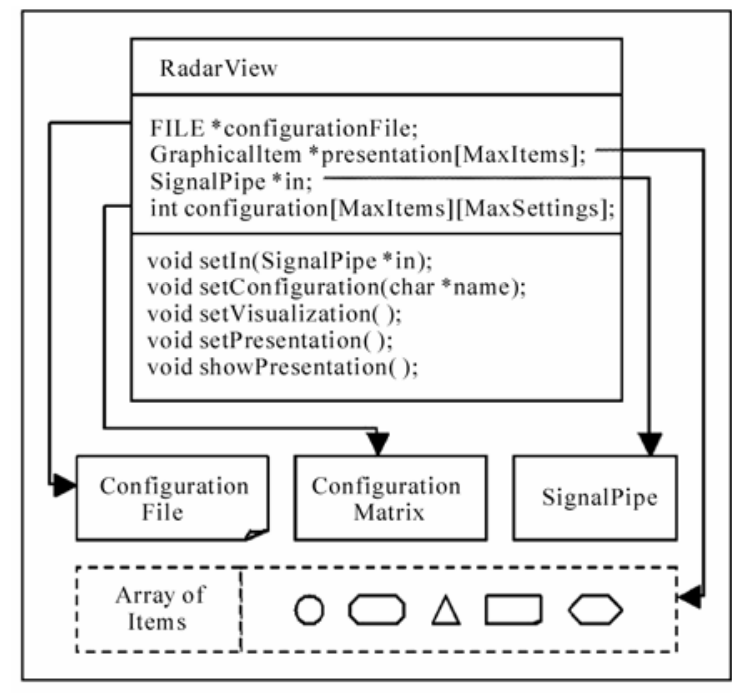

Figure 10. Implementation diagram of the class «RadarView»

The graphical items are stored in an array called «Presentation» (Figure 6) defined from the configuration matrix, and as the data of the updating of the different graphical items are modified to provide an inner representation of the view to be presented in the MFD (changes in the location of a contact or in the mode of operation of the radar).

\subsection{View Implementation}

A presentation is composed of a set of objective elements called "Graphical Items". Each item is represented as an instance of the abstract class «GraphicalItem». As many of the elements to be presented on the MFD are textual information, an abstract class which defines the specific behavior of a textual graphicable item called «GText».

The class «GraphicalItem» defines the behavior and properties characterizing an element of the presentation. To model a presentation it is necessary to design a class for each type of item that will compose it and these must be sub classes of «GraphicalItem». Thus, each sub class implements the behavior defined by the super class and a unified mechanism of treatment of the different graphicable elements is obtained.

Each graphicable item is defined by a set of attributes common to all of them (name, position in the axis X, axis $\mathrm{Y}$, colour) whose values are initialized from the format of representation set in the configuration matrix and they are updated in real time from the information of changes coming from the interpretation component.

\subsection{View Configuration}

The aim of configuration mechanism through files is to offer flexibility to handle the presentation formats. This mechanism allows the system to receive all the necessary 


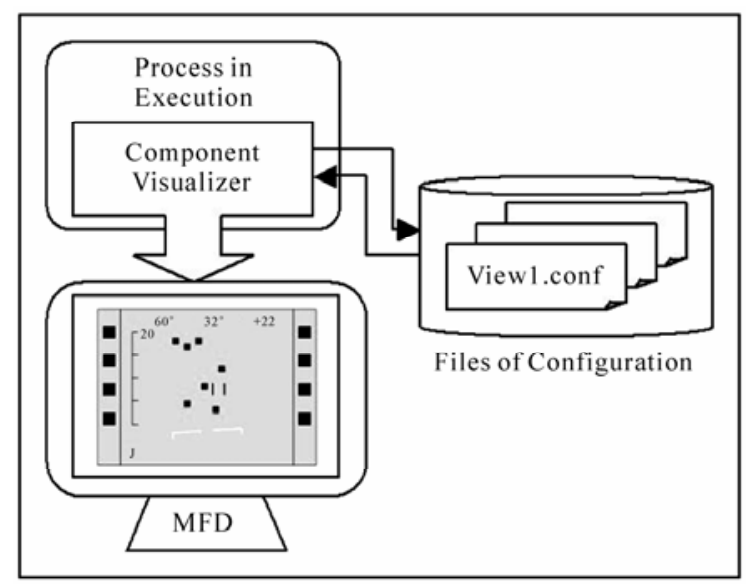

Figure 11. Diagram of interaction between files of configuration and the system

information to display a presentation on the screen from a file independent from the system (Figure 11). Thus, it is possible to modify the system through the configuration files with no need to recompile the code.

For each representation it is necessary to code an associated configuration file that includes a clear definition of the graphicable item including a predetermined presentation and the values corresponding to its inner attributes.

These items of the presentations enable to define views according to specific needs of each application domain. For example, an item to be defined in each presentation is its background which can be a color, a map, or a satellital photograph both for a air traffic radar and a meteorological radar. The system also provides the ability to present on screen textual information in different typographies and sizes, elemental graphs and images in different formats to identify a certain objective by means of a graphic representation.

\section{Fine Tuning For Real Radars}

As a case study a system previously described was implemented. The software system has been completely programmed in ANSI/C, and because of the characteristics for handling processes and real time services, it has been implemented on Linux operating system. The circuits and drivers have been completely specified by using VHDL on Xilinx platform.

\subsection{Signals Acquisition}

The technological platform used for the development was mainly based on a PC orienting the design to the definite implementation on a PC/104. As some recognition algorithms can not be implemented in software because of the performance requirements, coprocessors to implement these functions on FPGA were developed.

Additional boards with drivers for two devices of analogical and digital signal acquisition were added to the
PC. The former is a PCI board made by Measurement Computing, model PCI-DAS-4020/12 (Figure 12(a)). The latter is a PCI board made by Eagle Technology; model PCI-703S (Figure 12(b)). The PCI-DAS-4020/12 has 4 channels for analogical input, 2 channels for analogical output and 24 channels for digital I/O, with a resolution of 12 bit samples at a frequency of up to 80 Mhz. The PCI-703S has 8 channels for analogical input, 2 channels for analogical output and 8 channels for digital I/O, with a resolution of 14 bits samples at a frequency of up to $400 \mathrm{Khz}$.

The complex signal acquisition is made through FPGAs which realize a preprocessing to filter the analogical pulses and to recognize useful signal patterns. The FPGAs used are: Virtex-4 XC4VLX25 (Figure 13(a)) of Xilinx [11] of 24192 equivalent cells, $168 \mathrm{~KB}$ of RAM, $448 \mathrm{I} / \mathrm{O}$ pins and 48 DSPs which enables the operation as an analogical/digital conversor of signals of up to 105 Mhz with 14 bits of resolution per sampling [12]. The other FPGA is the Virtex-2 PRO XC2VP30 of Xilinx of 30816 equivalent cells, $428 \mathrm{~KB}$ of RAM and $644 \mathrm{I} / \mathrm{O}$ pins (Figure 13(b)).

\subsection{Signal Processing}

The algorithms of signal interpretation are realized on the two working platforms, both on the $\mathrm{PC}$ and by electronic circuits of specific application (FPGA) operating as preprocessors.

All the operations previous to the visualization, noise filtering, identification of possible objectives and calculus

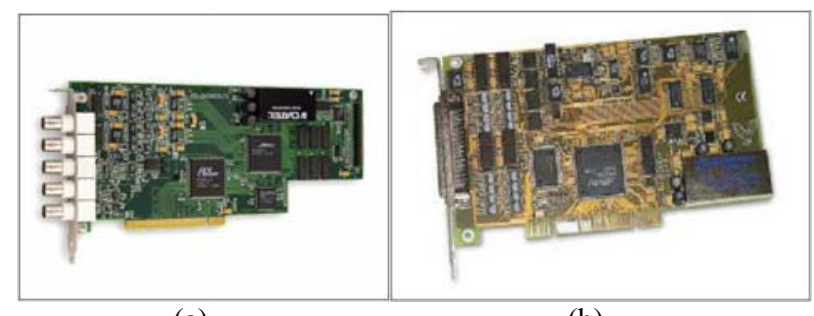

(a)

(b)

Figure 12. PCI-DAS-4020/12 (a) and PCI-703S (b)

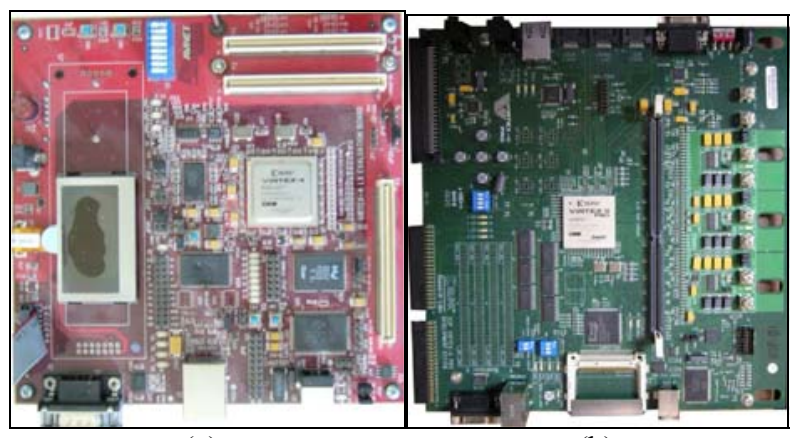

(a)

(b)

Figure 13. Left Virtex-4 XC4VLX25 (a) and Right Virtex-2 PRO XC2VP30 (b) 
of distances from the identified objectives are realized on the PC.

Owing to the high frequency of sampling of some signals and the high cost of processing in real time, it is necessary the inclusion of dedicated hardware for the processing of signals such as video, synchronisms, telemetry marks (tracking) and others. These signals provide the system with detailed information of targets, times and distances with precision of the order of microseconds. Such detailed analysis can not be made through software algorithms on the PC owing to the real time requirements.

The analysis and interpretation by hardware is materialized on devices of programmable logic or FPGAs that enable the synthesis of dedicated hardware circuits programmed in VHDL. The designed component behaves as a dedicated coprocessor of signals.

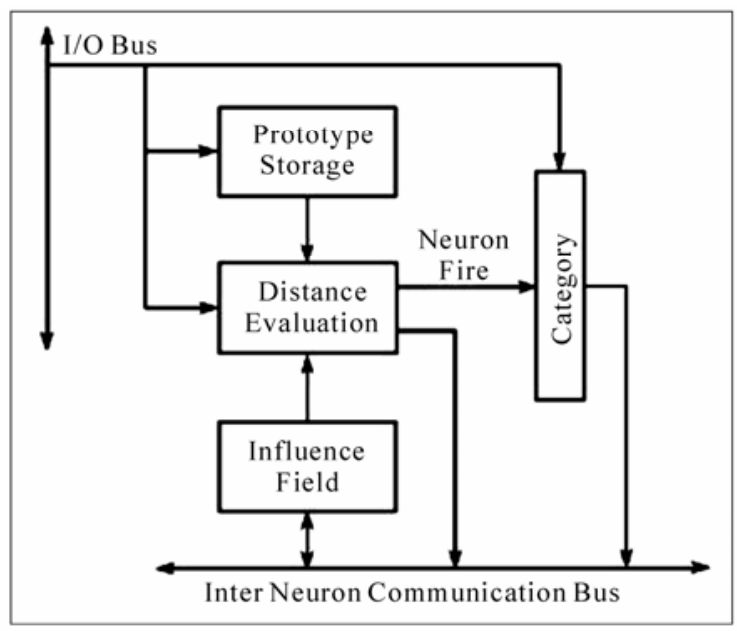

(a) Neuron structure

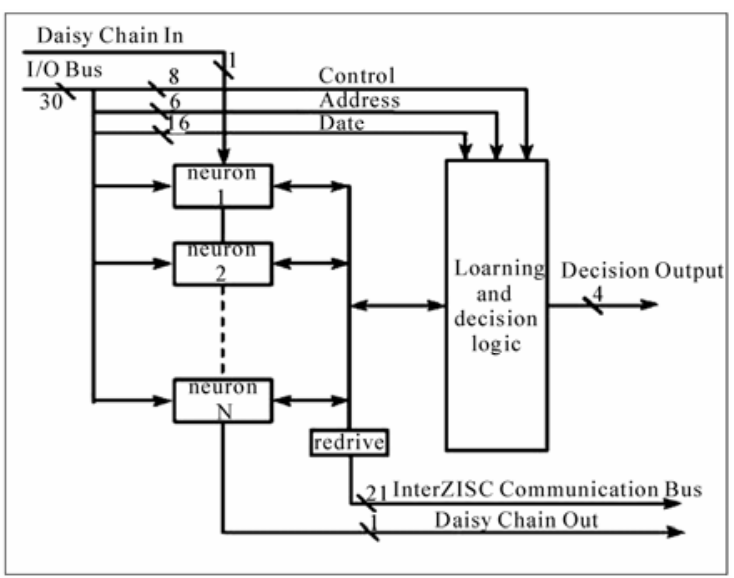

(b) Interconnection among neurons

Figure 14. Neuron structure and interconnection among neurons
This subsystem provides functions such as target recognition on a radar echo, search for determined targets in zones of radar echo (in a determined range of distance) and position of the telemetry mark. This function is implemented on dedicated hardware because it is a shortamplitude high-frequency pulse whose detection through software is complex and expensive.

Target recognition is implemented from a simplified and modified version of a Radial Basis Function Neural Networks (RBFNN) based on the neuronal processor ZISC78 [4-6,13-15] of IBM (Figure 14) which detects similarities between the information from the radar echoes and a variable set of training patterns representing the profiles of different objectives on the radar.

In this neuronal architecture each pattern representing a wave profile of an object is stored as a prototype in each neuron. Evaluating all the neurons in parellel the one having the prototype with a certain margin of similarity reacts sending a code to the controller of the net representing the belonging of the pattern to a determined category. Representing with characteristic prototypes the different objects to be recognized it is possible to detect them in each radar echo.

\subsection{Data Visualization}

Different presentations have been programmed and put into practice. Most of them are variations of the presentations type B which locate on the display different identified objectives on a cartesian representation according to the information of distance (axis Y) and azimuth (axis X) (Figure 15(a)). Presentations type A were also implemented, these show on the display a cartesian representation according to the information of amplitude of the signals received through the antenna (axis Y) and distance (axis X) [1] (Figure 15(b)).

\section{Acknowledgments}

We thank F. Oyarbide, F. Cutropía, L. Burgos, R. Cela, and C. Mayol, Redimec SRL staff. We are also grateful for the financial collaboration of Redimec SRL, National Agency of Scientific and Technological Promotion through the ANR 111/03 granted to Redimec SRL for the development of part of this project, Commission of Scientific Investigations of Buenos Aires Province for the scholarship to Martín F.Mezzanotte and all the technical staff of the institutions that have allowed us to work with their equipment.

\section{Conclusions}

Based on the tests made it is possible to conclude that the system developed can be successfully applied to different systems of existing radars to implement changes, updatings or improving with different adaptation grades and costs. This software was used on a Panel PC (Figure 16). [16]. 


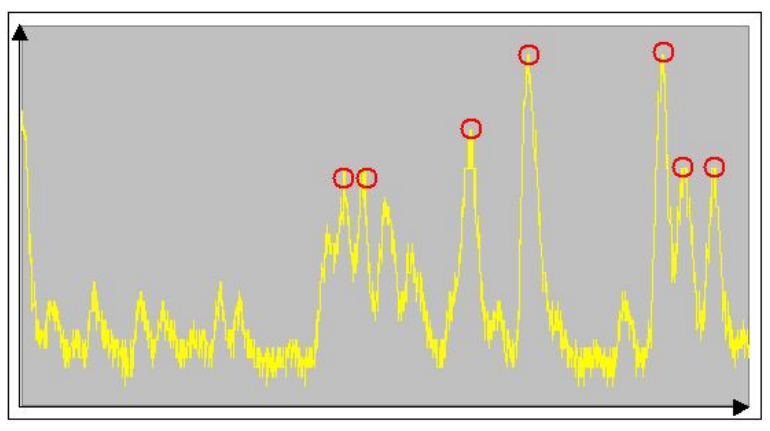

(a): Presentation "A"

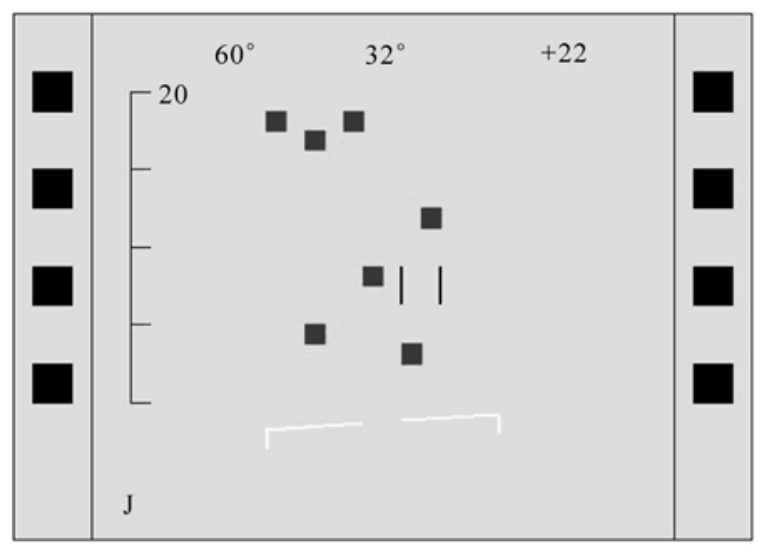

(b): Presentation "B"

Figure 15. Two kinds of radar displays
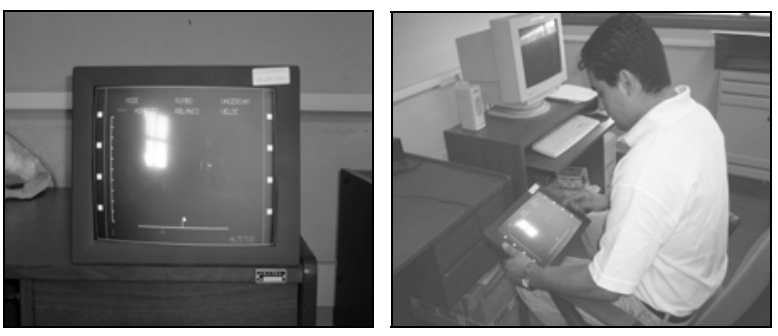

Figure 16. System tested on Panel PC with a LCD 12.1" TFT

The proposed architecture proved satisfactory to guarantee the fullfillment of functional and nonfunctional requirements. Anyway it is critical to apply this system on different technological platforms to test the functionalities in different application domains.

In a short time it is important to implement different signal processing algorithms. First, automatic recognition and tracking of objectives and/or paths algorithms must be developed.

The development of new presentations is also important since allows to adapt the present system to new and different application domains.

\section{REFERENCES}

[1] S. A. Hovanessian, "Radar detection and tracking systems," Artech House, Inc., 1973.

[2] L. Sha, et al., "Evolving dependable real-time systems," Technical Report CMS/SEI95 -TR-005, CMU, Software Engineering Institute, 1995.

[3] J. R. Y. Johnson and R. W. Johnson, "Challenges of computing the fast fourier transform," Optimized Portable Application Libraries (OPAL) Workshop, Kansas City, Junio de 1997.

[4] G. Noone, "Radar pulse train parameter estimation and tracking using neural networks, in proc," IEEE ANNES'95, IEEE, November 1995.

[5] E. Mingolla, W. Ross, and S. Grossberg, "A neural network for enhancing boundaries and surfaces in synthetic aperture radar images," Neural Networks 1999; 12 499-511.

[6] R. C. Krishnamohan and P. S. Mmoharir, "Radar signal design problem with neural network processing," Sadhana, Vol. 26, Part 3, pp. 237-241, June 2001.

[7] R Perry, et al., "Trellis structure approach to multitarget tracking. adaptive sensor array processing workshop," Marzo de 1999, MIT Lincoln Laboratory.

[8] G. Welch and G Bishop, "An introduction to the Kalman filter," Technical Report: TR95-041, University of North Carolina, 1995.

[9] Q. Zhao and Z. Bao, "Radar target recognition using a radial basis function," Neural Networks, Vol. 9, No. 4, pp. 709-720, Elsevier, 1996.

[10] Xilinx, Inc., Virtex-4 User Guide V2.2, www.xilinx.com, 2007.

[11] Xilinx, Ltd., Virtex-4 Family Overview, DS112 (V2.0), January 23, 2007.

[12] K. Chapman, Pico Blaze-Amplifier and A/D converter control for Spartan-3E Starter Kit, Xilinx Ltd, February 23, 2006.

[13] Solanki Gautam V., Neural network and its application in pattern recognition, Seminar Report, Indian Institute of Technology, 2004.

[14] Y. H, Liao, Neural networks in hardware: a survey, Department of Computer Science, University of California, 2001.

[15] Silicon Recognition, 1150 Industrial Avenue, Suite C, Petaluma, CA 94952, URL: www.silirec.com.

[16] Acosta et al., "Desarrollo de un Visualizador de Señales de Radar," CACIC, October 2006, San Luis, Argentina. 